\title{
Primary headache in the elderly in South-East Asia
}

\author{
Mei-Ling Sharon Tai · Jaishree Sharmini Jivanadham • \\ Chong Tin Tan $\cdot$ Vijay K. Sharma
}

Received: 7 January 2012/ Accepted: 1 March 2012/Published online: 16 March 2012

(c) The Author(s) 2012. This article is published with open access at Springerlink.com

\begin{abstract}
Headache aetiology and presentation are considerably different in elderly individuals. However, literature on headache characteristics among Asians is limited. The objective of this study was to evaluate the headache characteristics among elderly in an outpatient clinic setting in Malaysia, a South-East Asian country with diverse ethnicity. In this prospective cross-sectional study, patients presenting with headache to Neurology and Primary Care Clinics of University Malaya Medical Centre between February 2010 and July 2010 were included. Data for consecutive eligible adult patients were entered in a prospective headache registry. International Headache Criteria II (ICHD-II) were used to classify various headache subtypes. Patients with headache due to intracranial space occupying lesions were excluded. Patient were divided into two age groups-elderly (55 years and above) and younger (less than 55 years of age). Of the 175 screened patients, 165 were included in the study -70 in elderly age group and 95 in younger group. Tension-type headache was the commonest subtype (45.7 \%) among the elderly while Migraine without aura $(54.7 \%)$ was more common in young adults. More elderly patients suffered from chronic daily headache as compared to younger patients (47.1 vs. $28.4 \%$; $p=0.015)$. Headache subtypes and frequency differ considerably among elderly South East Asian patients.
\end{abstract}

M.-L. S. Tai $(\varangle)$ · J. S. Jivanadham · C. T. Tan Division of Neurology, Department of Medicine, University of Malaya, 50603 Kuala Lumpur, Malaysia e-mail: sharont1990@gmail.com

V. K. Sharma

Division of Neurology, National University Health System,

Singapore, Singapore
Keywords Headache $\cdot$ Elderly $\cdot$ Young $\cdot$ Clinic $\cdot$ Ethnic

\section{Introduction}

Headache is one of the most common conditions seen in general medicine as well as neurology specialist clinics [1]. Prevalence of headache in general population during 1 year has been reported as about $46 \%$ with the life-time prevalence of $64 \%$ [2]. Headache among the elderly has been evaluated only by a few studies [1,3-7]. Although, the prevalence of headache decreases with age $[3,8]$, it still remains a common complaint in the elderly $[8,9]$. Most of the previous studies on headache in the elderly have included Caucasian patients. Compared to males, females tend to have more headaches in all age groups [8-10]. Accordingly, among the elderly aged 75 years or more, the prevalence of headache was $55 \%$ in females compared to $22 \%$ males [8,9]. Expectedly, higher prevalence rates were reported among younger population with female preponderance $(66 \%$ females and $55 \%$ males in patients aged 55-74 years; $92 \%$ females and $74 \%$ males in younger adults aged 21-34 years) [8-10]. Although, headache starts at younger ages, de novo appearance in elderly may be observed in $5.4 \%$ patients aged 65 years old and above [6]. Furthermore, all subtypes of headache are observed among elderly. However, migraine occurs less frequently (4.6\%) as compared to tension-type headache $(16-27 \%)[5,8]$.

Various demographic and social factors influence the prevalence of headache. Accordingly, the prevalence of headache in rural Ecuador was found to be considerably lower (6.8 \% among elderly) as compared to the Western literature [11]. Data for headache among the elderly in Asia are scarce. In a study conducted in a nursing home in Thailand, 1-year headache prevalence of $55 \%$ was similar 
to Western studies [1]. However, a community study on Chinese elderly patients in Taiwan reported a relatively lower prevalence $(38 \%)$ of headache, with 1-year prevalence of tension-type headache in $20 \%$ of men and $46 \%$ of women [12]. Migraine was much less common and occurred in only $0.7 \%$ in men and $4.7 \%$ in women in this study [12]. Similar patterns of lower prevalence of migraine among the elderly were observed in other community studies $(0.27-0.76 \%$ and $0.3 \%$ in China and Hong Kong, respectively) [13-15].

While it is true that community-based study on headache is essential, it is equally important to study the characteristics of headache presenting to the clinic. This is because these are the headaches that burden the patient, which may be different from those ascertained by community based surveys. For example, a study from Denmark showed that $16 \%$ of migraine patients as compared to only $4 \%$ tension-type headache sought treatment from one or more specialists [16].

Malaysia is a developing country located in South East Asia. Its diverse ethnic distribution (consisting mainly of Malays, Chinese and Indians) may be considered a representative sample of Asian populations. There has been no previous clinic based study on frequency, characteristics and classification of headache among the elderly from Asia. We aimed to evaluate the characteristics of headache among the elderly in our Malaysian patients attending the outpatient clinics affiliated to our tertiary care center. We looked at the differences in the treatment seeking behavior for headache in our study cohort.

\section{Methods}

\section{Patient selection}

This was a prospective cross-sectional study done at the University Malaya Medical Centre between February 2010 and July 2010. The study was approved by the Institutional Ethics Committee of University Malaya Medical Centre. Consecutive patients, aged 18 years and more were recruited from the affiliated primary care and specialist neurology outpatient clinics. Patients referred to the neurology clinic and primary care clinic for headache were included in the study. All newcomers were also interviewed for the presence of headache. We evaluated the 3 -month prevalence of headache in our study population. Patients with headache secondary to intracranial mass lesions were excluded. Informed consent was obtained from all the study participants or their legally acceptable representatives. Patients were divided into two groups: aged 18-54 years (called younger patients) and those aged
55 years and above (called elderly). The age of 55 years chosen as cut-off to identify elderly was based on the mandatory retirement age in Malaysia of 55 years [17]. Furthermore, selecting a higher cut-off age would have adversely affected our recruitment rates due to lower prevalence of headache in patients more than 55-60 years old [7].

\section{Study design}

The study involved use of a structured headache questionnaire. The International Headache Society (IHS) classification, International Headache Criteria II (ICHD-II) were used to classify the headache subtypes [18]. Neuroimaging studies of the brain and/or cervical spine were performed, when necessary. All the patients were followed up in the neurology clinic after 1-5 months. Eleven patients unable to come to the clinic, due to various reasons, were interviewed through telephone.

\section{Data collection}

We collected information about the demographic characteristics, marital status, significant past medical history and various questions related to the headache profile. Specific data collected about headache included the time of onset, frequency, site, character and intensity. We defined chronic daily headache $(\mathrm{CDH})$ as a headache frequency more than 15 days in a month, with duration of more than $4 \mathrm{~h}$ a day [19]. The diagnosis of $\mathrm{CDH}$ and its subtypes was made on the initial interview. Use of various medications for pain relief was also assessed. We collected information about various non-pharmacological measures adopted by the patients during headache that included avoidance of certain food items that precipitated headache, use of wet towel or cold pack over head, avoiding the sun, lying quiet, tying a tight cloth around the forehead, traditional head and neck massage and local application of use of medicated oil. Data on trigger factors of headache such as stress, sun exposure, sleep deprivation, oversleep, missing meal and weather were also collected.

\section{Case definition}

The diagnosis of various subtypes of headache was based on the International Headache Society (IHS) Criteria (ICHD II) [18]. Briefly, the more common types of headache were: migraine with aura (Typical aura with migraine headache), migraine without aura, chronic migraine, infrequent episodic tension-type headache, frequent episodic tensiontype headache, chronic tension-type headache, cluster headache, trigeminal neuralgia and unspecified headache. 
Statistical analysis

All descriptive statistics were done using Statistical Package for Social Sciences, SPSS (Version 12.0, SPSS Inc., Chicago, USA). For categorical data, Chi-square test or Fisher's test was used. Continuous variables were expressed as means and analyzed with Student's $t$ test. A $p$ value of $<0.05$ was taken as statistically significant.

\section{Results}

Out of the total of 1,020 patients who attended the participating outpatient clinics during the project, 175 $(17.2 \%)$ met our inclusion criteria of having history of headache at least once per month for more than 3 months. Finally, 168 patients were found to be eligible for the study; $90 \%$ of the patients were recruited from neurology clinic and $10 \%$ of the patients were from primary care clinic. The main reasons for exclusion were age less than 18 years old (5 cases) and intracranial mass lesion ( 2 cases). Three patients withdrew consent, leaving 165 cases for final analysis.

\section{Demographic characteristics}

Of the 165 patients included in the final analyses, 70 $(42.4 \%)$ were elderly (mean age $64.6 \pm 7.6$ years) and 95 $(57.6 \%)$ were younger adults (mean age $29.8 \pm 6.4$ years). For the elderly patients, 32 patients were aged 55-64 years (45.7\% of all elderly patients), 32 in age group 65-74 years (45.7\% of all the elderly patients) and six aged $75-84$ years (8.6 \% of total elderly patients). Table 1 lists the demographic characteristics of the study population. Briefly, there were more female patients compared to male patients in both age groups. There was a fair representation of all major ethnicities in both age groups.

In the elderly, two patients had onset of migraine without aura, whereas one patient had onset of migraine with aura at $\geq 55$ years old. Fifteen patients had onset of tension-type headache, two had trigeminal neuralgia and ten had unspecified headache at 55 years old and above. The age of onset for cluster headache was 61 years old whereas for glossopharyngeal neuralgia, onset of headache was 67 years old. Overall, $46 \%$ of the elderly patients had onset of headache at $\geq 55$ years old and headache starting at that age represented $19 \%$ of headache in all ages.

In the young adults, the age of onset for migraine without aura was 10-39 years old, migraine with aura 11-34 years old, tension-type headache 10-38 years old, cluster headache 30-35 years old, trigeminal neuralgia 37 years old and unspecified headache 10-30 years old.
Table 1 Demographic characteristics of study population $(n=165)$

\begin{tabular}{llll}
\hline Variable & $\begin{array}{l}\text { Young } \\
(n=95)\end{array}$ & $\begin{array}{l}\text { Elderly } \\
(n=70)\end{array}$ & $p$ value \\
\hline $\begin{array}{l}\text { Age }(\text { mean } \pm \mathrm{SD}) \\
\text { Gender }(n, \%)\end{array}$ & $29.8 \pm 6.4$ & $64.6 \pm 7.6$ & $<0.0001$ \\
$\quad$ Male & $27(28.4)$ & $24(34.3)$ & 0.5 \\
Female & $68(71.6)$ & $46(65.7)$ & \\
Ethnic groups $(n, \%)$ & & & \\
Malay & $54(56.8)$ & $16(22.9)$ & $<0.0001$ \\
Chinese & $12(12.6)$ & $27(38.6)$ & $<0.0001$ \\
Indian & $25(26.3)$ & $26(37.1)$ & 0.17 \\
Others & $4(4.3)$ & $1(1.4)$ & \\
BMI $\left(\mathrm{kg} / \mathrm{m}^{2}\right)$ & $25.24 \pm 5.92$ & $25.92 \pm 4.78$ & 0.44 \\
$\quad($ mean \pm SD) & & & $<0.0001$ \\
Marital status $(n, \%)$ & & $7(10.0)$ & 0.007 \\
Single & $48(50.5)$ & $46(65.7)$ & $<0.0001$ \\
Married & $42(44.2)$ & $15(21.4)$ & 0.7 \\
Widow/widower & 0 & $2(2.9)$ & \\
Divorced/separated & $5(5.3)$ & & \\
\hline
\end{tabular}

Headache characteristics

Headache characteristics in the two age groups in our study population are represented in Table 2 . Briefly, more elderly patients suffered from headaches everyday compared to the young (41.4 vs. $14.7 \% ; p<0.0001)$. Headache on more than 15 days in a month occurred more frequently among the elderly as compared to the younger patients (47.1 vs. $28.4 \% ; p=0.015$ ). Headache of recent onset was noted in three elderly (two with unspecified headache and one with episodic tension-type headache) and one younger patient (with episodic tension-type headache).

Temporal headaches were seen more commonly among the younger patients ( 49.5 vs. $25.7 \%$ in the elderly; $p=$ $0.002)$, largely due to the higher prevalence of migraine. Similarly, facial pain was more common among the elderly (10 vs. $1.1 \%$ in younger age; $p=0.01)$ due to higher prevalence of trigeminal neuralgia.

Types of headache according to ICHD II classification

Headache subtypes differed in the two age groups (Table 3). While migraine without aura (54.7\%) and migraine with aura $(16.8 \%)$ were more common among the younger patients, the commonest headache in the elderly was tension-type headache $(45.7 \%)$ and half of them were chronic in nature. Twelve young adults and four elderly patients suffered from chronic migraine. None of the patients fulfilled the diagnostic criteria of medicationoveruse headache. 
Table 2 Comparison of headache characteristics among young and elderly

\begin{tabular}{|c|c|c|c|}
\hline Variable & Young $(n=95)$ & Elderly $(n=70)$ & $p$ value \\
\hline \multicolumn{4}{|l|}{ Time of onset $(n, \%)$} \\
\hline On waking up & $14(14.7)$ & $25(35.7)$ & 0.003 \\
\hline Afternoon/evening & $21(22.1)$ & $15((21.4)$ & 1.0 \\
\hline Night & $7(7.4)$ & $2(2.9)$ & 0.3 \\
\hline Anytime & $53(55.8)$ & $28(40)$ & 0.06 \\
\hline \multicolumn{4}{|l|}{ Frequency $(n, \%)$} \\
\hline Everyday & $14(14.7)$ & $29(41.4)$ & $<0.0001$ \\
\hline Every other day & $10(10.5)$ & $7(10)$ & 1.0 \\
\hline$>1-3 \times /$ week & $40(42.1)$ & $22(31.4)$ & 0.19 \\
\hline $1 \times /$ week $-1 \times / 2$ week & $15(15.8)$ & $3(4.3)$ & 0.02 \\
\hline$<1 \times / 2$ week $-1 \times /$ month & $7(7.4)$ & $5(7.1)$ & 1.0 \\
\hline$<1 \times /$ month $-1 \times / 6$ months & $7(7.4)$ & $1(1.4)$ & 0.14 \\
\hline$<1 \times / 6$ months $-1 \times /$ year & $2(2.1)$ & $3(4.3)$ & 0.65 \\
\hline \multicolumn{4}{|c|}{ Number of headache in a month $(n, \%)$} \\
\hline Headache $\leq 15$ days/month & $68(71.6)$ & 37 (52.9) & 0.015 \\
\hline Headache $>15$ days/month & $27(28.4)$ & $33(47.1)$ & 0.015 \\
\hline \multicolumn{4}{|l|}{ Site of pain $(n, \%)$} \\
\hline Frontal & $12(12.6)$ & $9(12.9)$ & 1.0 \\
\hline Temporal & $47(49.5)$ & $18(25.7)$ & 0.002 \\
\hline Occipital & $16(16.8)$ & $20(28.6)$ & 0.09 \\
\hline Vertex & $11(11.6)$ & $7(10)$ & 0.81 \\
\hline Whole head & $6(6.3)$ & $5(7.1)$ & 1.0 \\
\hline Migrating & $1(1.1)$ & $1(1.4)$ & 1.0 \\
\hline Face & $1(1.1)$ & $7(10)$ & 0.01 \\
\hline Back of neck & $1(1.1)$ & $3(4.3)$ & 0.31 \\
\hline \multicolumn{4}{|l|}{ Character of headache $(n, \%)$} \\
\hline Throbbing/pulsating & $61(64.2)$ & $32(45.7)$ & 0.02 \\
\hline Sharp/stabbing & $19(20.0)$ & $12(17.1)$ & 0.69 \\
\hline Tightness/pressing & $14(14.7)$ & $18(25.7)$ & 0.11 \\
\hline Not specific & $1(1.1)$ & $8(11.4)$ & 0.005 \\
\hline \multicolumn{4}{|l|}{ Intensity of headache $(n, \%)$} \\
\hline Mild & $9(9.5)$ & $28(40)$ & $<0.0001$ \\
\hline Moderate & $45(47.4)$ & $19(27.1)$ & 0.01 \\
\hline Severe & $41(43.2)$ & $23(32.9)$ & 0.19 \\
\hline
\end{tabular}

Table 3 Headache classification according to age group

\begin{tabular}{llll}
\hline Headache classification & Young $(n=95, \%)$ & Elderly $(n=70, \%)$ & $p$ value \\
\hline Migraine without aura & $52(54.7)$ & $9(12.9)$ & $<0.0001$ \\
Migraine with aura & $16(16.8)$ & $4(5.7)$ & 0.032 \\
Chronic migraine & $12(12.6)$ & $4(5.7)$ & 0.19 \\
Infrequent and frequent episodic & $11(11.6)$ & $16(22.9)$ & 0.06 \\
$\quad$ tension-type headache & $5(5.3)$ & $16(22.9)$ & 0.002 \\
Chronic tension-type headache & $2(2.1)$ & $1(1.4)$ & 1.0 \\
Cluster headache & $1(1.1)$ & $5(7.1)$ & 0.08 \\
Trigeminal neuralgia & $0(0)$ & $1(1.4)$ & 0.424 \\
Glossopharyngeal neuralgia & $8(8.4)$ & $18(25.7)$ & 0.004 \\
Unspecified headache & &
\end{tabular}


As for $\mathrm{CDH}$, five out of 16 elderly patients with chronic tension-type headache had the onset aged less than 55 years old. Headache onset in three out of four elderly patients with chronic migraine occurred before 55 years of age.

Trigger factors for the headache

Sun exposure was the most common trigger in both age groups, followed by sleep deprivation, weather, missing meal and stress.

Treatment seeking behavior for the headache

We did not observe any significant difference in the treatment seeking behavior for headache in the two age groups. Overall, avoidance of food items that precipitated headache such as chocolates and coffee was widely practiced by both age groups and head-massaging was the commonest method used to relieve the headache.

Avoidance of food which precipitated headache was practiced by more migraine patients in the elderly and young adults. Five elderly patients who had migraine without aura, two patients with migraine with aura, two with tension-type headache, four with unspecified headache, one with glossopharyngeal neuralgia and one with trigeminal neuralgia avoided food which precipitated headache. In the younger group, avoidance of food items that precipitated headache was practiced by six patients with migraine with aura, 13 with migraine without aura, two with tension-type headache, two with unspecified headache and one patient with trigeminal neuralgia.

Head-massaging was practiced by more elderly and younger patients with tension-type headache. Head-massaging was used by nine patients with tension-type headache and two with unspecified headache in the elderly group. In the younger group, massaging was used by six patients with tension-type headache, five with migraine without aura, two with migraine with aura and two with unspecified headache.

\section{Discussion}

Our study describes headache characteristics, subtypes and frequency among the young and elderly patients attending outpatient primary care and specialist clinic affiliated to our tertiary care center in Malaysia. Some of the headache features showed significant differences between the young and the elderly age groups. Despite significant differences in headache characteristics and subtypes, the treatment seeking behavior of the elderly patients were similar to the younger adults. Malaysia is a developing nation. Owing to a good mixture of the three major ethnicities (Chinese, Indians and Malays) in the region, our study population might be considered a good representative of South-East Asia.

Headache is a common disease among Malaysians with 1 year prevalence of $26.5 \%$ for tension-type headache, $9.0 \%$ for migraine and $28.2 \%$ for other subtypes of headache [20]. In our study, $42.4 \%$ patients were elderly (mean age $64.6 \pm 7.6$ years). Our findings are consistent with the previous community-based study in Malaysia by Alders et al. [20] that reported the prevalence of headache in various age groups; $46 \%$ in patients before 15 years of age, $80 \%$ in age group $16-25$ years, $78 \%$ in age group $26-35$ years, $73 \%$ in age group 36-45 years, $54 \%$ in age group $46-55$ years and $39 \%$ in those more than 65 years old.

A significant proportion of elderly patients in our study suffered from chronic daily headaches $(47.1 \%)$ while the headache frequency was lower (less than 15 days/month) among the younger $(71.6 \%)$ patients. Perhaps, this might be explained by the relatively greater frequency of chronic tension-type headache in the elderly as compared to the migraine headache in the young. Our observations are consistent with the study by Langemark et al. [21] in which chronic headaches were frequent among the elderly, affecting about $10 \%$ of women and $5 \%$ of men above 70 years. Similar findings have been reported among the elderly in various community studies from Italy, Taiwan and Georgia [4, 22, 23].

A sizeable minority of the elderly patients that had CDH had such headaches starting at a younger age. This feature has been reported consistently in various population studies $[23,24]$. Furthermore, these studies have reported the lack of decline in $\mathrm{CDH}$ prevalence. Interestingly, a recent large population-based study reported that despite the decline in the prevalence of episodic headache, the remission rates of chronic migraine are not lower in the elderly as compared to a younger age [24].

An important finding of our study is the considerably higher headache frequency among our elderly study participants. Most (94.3\%) of the Malaysian elderly headache patients reported at least one episode in a month, which is relatively much higher than the previously reported figures of $18.0 \%$ in Italians [4] and $33 \%$ among the elderly nursing home residents in Thailand (33\%) [1]. Although, difficult to substantiate, higher education level, cultural differences and physical independency could have contributed to the observed differences. The elderly patients with episodic headaches had an established pattern for a long time and did not seek care for headache. In comparison, the episodic headaches in the younger group were more likely to be of recent onset.

Another interesting feature of headache patients is the treatment seeking behavior in various age groups. While 
the elderly seek medical attention for tension-type headache for reassurance [25], younger patients are usually driven to the outpatient clinics due to the headache severity $[4,6,21]$. Although, we observed a similar pattern, this phenomenon occurred even when a larger proportion of study patients $(60 \%)$ presented with moderate-to-severe headache, which is comparatively higher than the prevalence of $20.4 \%$ in an Italian study by Prencipe et al. [4]. Perhaps, the observed difference is due to the largely rural population in the Italian study [4]. One distinctive observation in our study is the higher proportion of chronic tension-type headache among the patients with tension headache. This is in contrast to the Bruneck Study done in Italy that reported a lower $(35.8 \%) 1$ year prevalence of tension-type headache with only $5 \%$ patients having chronic tension-type headache [26]. This could have occurred due to the hospital based nature of our study as compared to the population-based Bruneck study [26]. Our study was conducted in a tertiary hospital located in the urban Malaysia with easy and relatively cheaper medical care and transport services and these logistic factors might have accounted for the higher number of headache patients seeking medical treatment [25].

The influence of culture on the treatment seeking behavior was evident in our study. Accordingly, the practice of head and neck massage and the use of medicated oil, a common tradition of South East Asia [27, 28] were widely practiced by our study participants. Interestingly, the treatment seeking behavior did not differ between the young and the elderly patients, probably reflecting the underlying concepts, cultural factors and traditional attitudes to the illness. Head-massaging was used more by our patients with tension-type headache.

Zanchin et al. [29] reported that massage on the temples and nape was the most common maneuver used by tensiontype headache patients.

Our study has certain limitations. First, our study might be considered to be small in size. Second, our study sample included largely an urban population attending the clinics affiliated to a tertiary center. The patients who attended the neurology clinic were referred patients. However, we believe that inclusion of consecutive and urban patients could have compensated for the small numbers by improving the quality of data.

In conclusions, we present the headache characteristics, subtypes and treatment seeking behavior among the multiethnic South-East Asian patients. Our findings would contribute to the limited information available on headache among Asians in general and among the elderly in particular. Perhaps, a larger Asia-wide study on headache is warranted to understand the contributions of ethnic/racial factors in the field of neurology of headache.
Conflict of interest The authors declare no conflict of interest.

Open Access This article is distributed under the terms of the Creative Commons Attribution License which permits any use, distribution, and reproduction in any medium, provided the original author(s) and the source are credited.

\section{References}

1. Srikiatkhachorn A (1991) Epidemiology of headache in the Thai elderly: a study in the Bangkae Home for the aged. Headache 31:677-681

2. Manzoni GC, Stovner LJ (2010) Epidemiology of headache. Handbook of Clinical Neurology 97:3-22

3. Ward TN (2002) Headache disorders in the elderly. Curr Treat Options Neurol 4:403-408

4. Prencipe M, Casini AR, Ferretti C, Santini M, Pezzella F, Scaldaferri $N$ et al (2001) Prevalence of headache in an elderly population: attack frequency, disability, and use of medication. J Neurol Neurosurg Psychiatry 70:377-381

5. Camarda R, Monastero R (2003) Prevalence of primary headache in Italian elderly: prelimanary data from the Zabut aging project. Neurol Sci 24:122-124

6. Pascual J, Berciano J (1994) Experience in the diagnosis of headaches that start in the elderly. J Neurol Neurosurg Psychiatry 57:1255-1257

7. Lisotto C, Mainardi F, Maggioni F, Dainese F, Zanchin G (2004) Headache in the elderly: a clinical study. J Headache Pain 5:36-41

8. Capobianco DJ (2003) Headache in the elderly. Advanced studies in medicine 3(6C):S556-561

9. Mariecken Fowler V, David Capobianco J, David Dodick W (2004) Headache in the Elderly. Sem Pain Med 2:123-128

10. Waters WE (1974) The Pontypridd headache survey. Headache 14:81-90

11. Sachs H, Sevilla F, Barberis P, Bolis L, Schoenberg B, Cruz M (1985) Headache in the rural village of Quiroga, Ecuador. Headache 25:190-193

12. Wang SJ, Liu HC, Fuh JL, Liu CY, Lin KP, Chen HM et al (1997) Prevalence of headaches in a Chinese elderly population in Kinmen: age and gender effect and cross-cultural comparisons. Neurology 49:195-200

13. Cheng XM, Cai K, Li SC, Wong WJ, Wu SP (1990) An epidemiologic survey of migraine in six cities in China. Chin J Neurol 23:44-46

14. Zhao F, Tsay JY, Cheng XM, Wong WJ, Li SC, Yao SX et al (1988) Epidemiology of migraine: a survey in 21 provinces of the People's Republic of China, 1985. Headache 28:558-565

15. Wong TW, Wong KS, Yu TS, Kay R (1995) Prevalence of migraine and other headaches in Hong Kong. Neuroepidemiology 14:82-91

16. Rasmussen BK, Jensen R, Olesen J (1992) Impact of headache on sickness absence and utilisation of medical services: a Danish population study. J Epidemiol Community Health 46(4):443-446

17. Masud J, Haron SA, Gikonyo LW (2008) Gender differences in income sources of the elderly in Peninsular Malaysia. J Fam Econ 29:623-633

18. ICHD-II International Headache Society Classification. http://ihsclassification.org/en/

19. Lu SR, Fuh JL, Chen WT, Juang KD, Wang SJ (2001) Chronic daily headache in Taipei, Taiwan: prevalence, follow-up and outcome predictors. Cephalalgia 21:980-986 
20. Alders EEA, Hentzen A, Tan CT (1996) A community-based prevalence study on headache in Malaysia. Headache 36:379-384

21. Langemark M, Olsen J, Poulsen DL, Bech P (1988) Clinical characterization of patients with chronic tension headaches. Headache 28(9):590-596

22. Katsarava Z, Dzagnidze A, Kukava M, Mirvelashvili E, Djibuti M, Janelidze M et al (2009) Primary headache disorders in the Republic of Georgia: prevalence and risk factors. Neurology 73: 1796-1803

23. Wang SJ, Fuh JL, Lu SR, Liu CY, Hsu LC, Wang PN et al (2000) Chronic daily headache in Chinese elderly: prevalence, risk factors, and biannual follow-up. Neurology 54:314

24. Manack A, Buse DC, Serrano D, Turkel CC, Lipton RB (2011) Rates, predictors, and consequences of remission from chronic migraine to episodic migraine. Neurology 76:711-718
25. Wang SJ, Fuh JL, Young YH, Lu SR, Shia BC (2001) Frequency and predictors of Physician consultations for headache. Cephalalgia 21:25-30

26. Schwaiger J, Kiechl S, Seppi K, Sawires M, Stockner H, Erlacher $T$ et al (2009) Prevalence of primary headaches and cranial neuralgias in men and women aged 55-94 years (Bruneck Study). Cephalalgia 29:179-187

27. Tan CT (1997) Cultural belief system and headache. Neurol J Southeast Asia 2:147-150

28. Buchwald D, Panwala S, Hooton TM (1992) Use of traditional health practices by Southeast Asian refugees in a primary care clinic. West J Med 156:507-511

29. Zanchin G, Maggioni F, Granella F, Rossi P, Falco L, Manzoni GC (2001) Self-administered pain-relieving manoeuvres in primary headaches. Cephalalgia 21:718-726 